\title{
A rare esophagus damage reason; Ulcerative esophagitis due to usage of doxycycline
}

\author{
Durmuş Ali Çetin, ${ }^{1}$ Mehmet Patmano, ${ }^{2}$ Tufan Gümüş ${ }^{2}$ \\ 'Department of Gastroenterological Surgery, Şanlıurfa Training and Research Hospital, Şanlıurfa, Turkey \\ 2Department of General Surgery, Şanlıurfa Training and Research Hospital, Şanlıurfa, Turkey
}

Dear Editor,

Esophagitis is a mucosa damage on the esophagus due related to various reasons. Medication-related esophagitis is a condition characterized by damage in the esophagus mucosa and ulceration following usage of various drugs. The most frequently implied drugs for this condition are doxycycline, tetracycline, non-steroid anti-inflammatory drugs, clindamycin, aspirin, potation chloride and alendronate sodium. ${ }^{[1,2]}$ Risk factors for esophagitis after usage of drugs are taking gelatin-coated pills and capsules without sufficient amount of fluids, the quantity of the drug, and sleeping right after taking the drug. [3] Typical symptoms of medication-related esophagitis are retrosternal burning that occurs after taking drugs, pain that extends to the back and odynophagia. Upper gastrointestinal system (GIS) endoscopy is the gold standard for diagnosis and monitoring of esophagitis that develops due to medication. The first step in treatment is stopping the intake of the drug that leads to esophagitis. Proton-pump inhibitors (PPI), H2 receptor blockers, antacids and sucralfate are recommended for supportive treatment. In this article, we aimed to present our case who developed ulcerative esophagitis after usage of doxycycline.
The thirty-year-old female patient started 2x100 mg doxycycline treatment 5 days ago due to her diagnosis of menstrual disorder and vaginitis at the gynecology polyclinic. She was admitted to our clinic with complaints of retrosternal burning, back pain and painful swallowing. Medication-related esophagitis came to mind for the patient because of the existing clinical signs and her history of medication. An upper GIS endoscopy was planned. In the upper GIS endoscopy procedure, we observed an area surrounded by hyperemic ulcer which covered $3 / 4$ of the esophagus lumen starting at the incisor teeth at around $27-28 \mathrm{~cm}$ (Fig. 1a, b). The laboratory values of the patient were in the normal interval. Doxycycline treatment was stopped. Oral intake of fluids and soft foods was achieved. Treatment was started with $1 \times 40 \mathrm{mg} /$ day of pantoprazole, $1 \times 40 \mathrm{mg} /$ day of famotidine, $4 \mathrm{x} 1 \mathrm{~g} /$ day of sucralfate. After monitoring the patient, it was seen that her complaints disappeared, and in the upper GIS endoscopy check after 1 month, the ulcerated area on the esophagus recovered.

Medication-related esophagitis was defined for the first time in the literature after usage of potassium chloride tablets. ${ }^{[4]}$ Later, it was reported that hundreds of different drugs caused esophagitis as etiologic agents. ${ }^{[5]}$ The drugs responsible for most of the cases are doxycycline and tetracycline. Various parameters of the drug, the person 

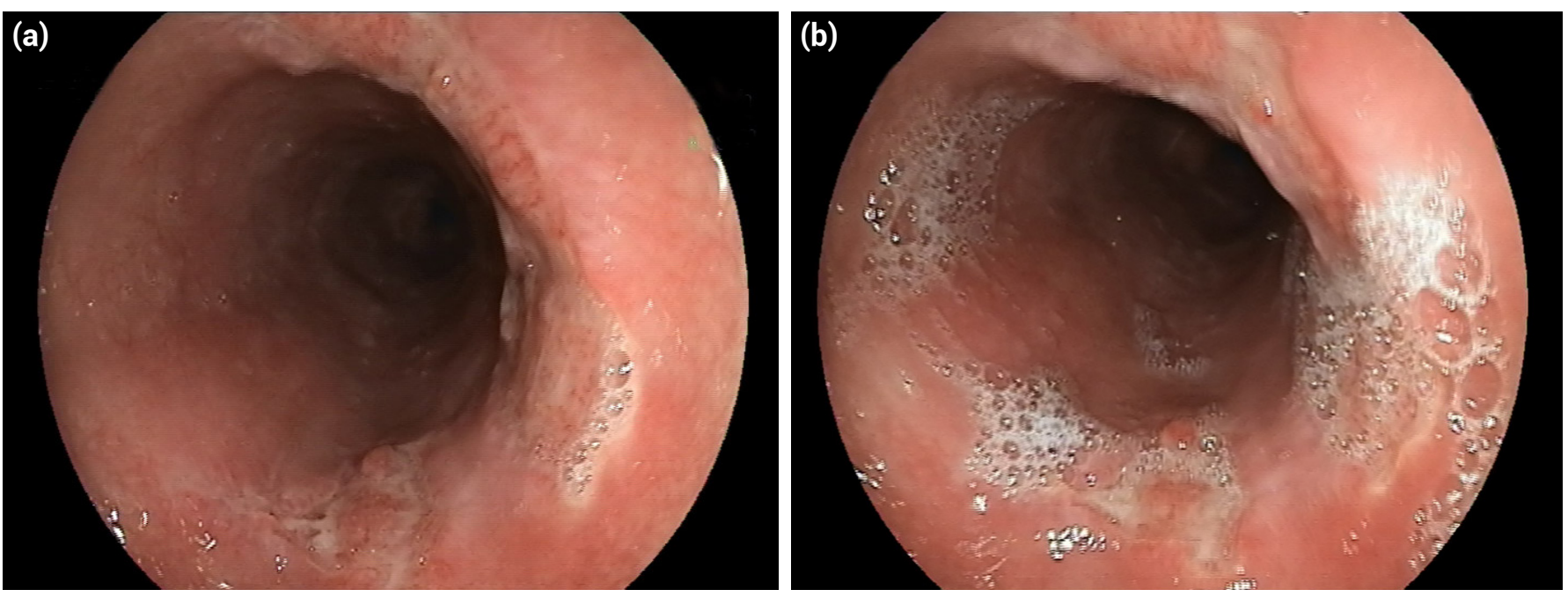

Figure 1. (a, b) Appearance of ulcerative esophagitis in upper GIS endoscopy.

and the esophagus are effective in the mucosa damage in the esophagus. The most important known factors for medication-related esophagitis are taking the drug without sufficient amount of fluids and sleeping right after taking the drug. ${ }^{[2]}$ Our patient stated in her history that she recently took the drug before sleeping with a small amount of water. Typical clinical signs are odynophagia, retrosternal pain-burning, dysphagia and epigastric pain that develop within hours or days. ${ }^{[2]}$ Upper GIS endoscopy is the most important method in the diagnosis, differential diagnosis and monitoring of esophagitis that develops due to medication. It was reported that medicationrelated esophagus damage is most frequently seen in the middle esophagus. The most frequently observed endoscopic signs in medication-related esophagus damage are one or more non-specific surface ulcers that are separate or clustered. Deeper ulcers that may lead to bleeding may rarely be encountered. It was reported that ulcers that develop because of doxycycline and tetracycline are small, separate from each other, and they are located in the middle esophagus. ${ }^{[1,2]}$ Our case was admitted with complaints of retrosternal burning, back pain and odynophagia. In the upper GIS endoscopy, an area surrounded by continuous hyperemic ulcers was observed to cover $3 / 4$ of the esophagus lumen. In terms of histopathological analysis, biopsy is not required for diagnosis. However, it may be required in lesions with malign appearance in differential diagnosis. The first step in the treatment of medication-related ulcerative esophagitis is stopping the intake of the drug that is causing the esophagitis. For the next step, PPIs, H2 receptor blockers, antacids and sucralfate are recommended. In patients with serious complaints, oral intake may be stopped, and intravenous fluid support may be required. In our case, firstly the doxycycline intake was stopped, and PPI, $\mathrm{H} 2$ receptor blocker and sucralfate treatment was administered.

Consequently, almost all types of drugs, especially doxycycline and tetracycline may lead to ulcerative esophagitis. Diagnosis of medication-related esophagitis may be made by upper GIS endoscopy along with a typical history and clinical findings. Doxycycline and tetracycline are used frequently by young and female patients for treatment of gynecological infections and acne vulgaris. The two most important fixable factors in medication-related esophagitis development is taking drugs with insufficient amounts of water and sleeping after a short time after taking the drug. If physicians are alert about the potential of such drugs to cause ulcerative esophagitis and they warn patients about the ways to use such drugs, cases of medication-related esophagitis may be prevented to a significant extent.

\section{References}

1. Abid S, Mumtaz K, Jafri W, Hamid S, Abbas Z, Shah HA, et al. Pill-induced esophageal injury: endoscopic features and clinical outcomes. Endoscopy 2005;37:740-4. [CrossRef]

2. Dağ MS, Öztürk ZA, Akın I, Tutar E, Çıkman Ö, Gülşen MT. Druginduced esophageal ulcers: case series and the review of the literature. Turk J Gastroenterol 2014;25:180-4. [CrossRef]

3. Vãlean S, Petrescu M, Cãtinean A, Chira R, Mircea PA. Pill esophagitis. Rom J Gastroenterol 2005;14:159-63.

4. Pemberton J. Oesophageal obstruction and ulceration caused by oral potassium therapy. Br Heart J 1970;32:2678. [CrossRef]

5. Kikendall JW. Pill esophagitis. J Clin Gastroenterol 1999;28:298-305. [CrossRef] 